\title{
Advantages of an Ornithopter versus an Airplane with a Propeller
}

\author{
By Shigeru SunADA and Kazuki TsuJI \\ Osaka Prefecture University, Osaka, Japan
}

(Received June 17th, 2012)

\begin{abstract}
The variation in aerodynamic forces acting on an ornithopter and an airplane with one or two propellers, which generate the same thrust under no gust wind, is compared when they encounter a gust of wind. The consumed power, or the period of one cycle of flapping motion and that of one rotation of propeller(s), remains constant before and after they encounter a wind gust. The following results are obtained under both conditions. The variation in aerodynamic forces caused by vertical and frontal wind gusts of an ornithopter are a little smaller than that of an airplane with one or two propellers. The difference in variation in aerodynamic forces caused by a side wind gust between them are often larger than that caused by vertical and frontal wind gusts. The variation in aerodynamic forces caused by a side wind gust of the former is smaller than that of the latter when the reciprocal of the advance ratio of the propeller and the flapping amplitude of the ornithopter are small.
\end{abstract}

Key Words: Ornithopter, Propeller, Gust

\section{Nomenclature}

$b$ : number of blades

$c$ : chord length of the main wing and beating wing

$c_{P}$ : chord length of the propeller

$C_{\ell}:$ lift coefficient

$C_{\ell \alpha}:$ lift slope

$\bar{D}_{F}$ : parasite drag with no wind gust

$\mathrm{d} L, \mathrm{~d} D$ : lift and drag acting on the wing element $F_{X}, F_{Y}, F_{Z}$ : aerodynamic forces caused by the right and left flapping wings in the $X, Y,-Z$ directions

$F_{X P}, F_{Y P}, F_{Z P}$ : aerodynamic forces caused by all propellers in the $X, Y,-Z$ directions

$F_{X W}, F_{Y W}, F_{Z W}$ : aerodynamic forces caused by the main fixed wing in the $X, Y,-Z$ directions

$\bar{F}_{X}, \bar{F}_{Z}$ : aerodynamic forces caused by the right and left flapping wings in the $X,-Z$ directions with no wind gust

$\bar{F}_{X P}$ : aerodynamic forces caused by all propellers in the $X$ direction with no wind gust

$\bar{F}_{X W}, \bar{F}_{Z W}$ : aerodynamic forces caused by the main fixed wing in the $X,-Z$ directions with no wind gust

$\tilde{F}_{X}, \tilde{F}_{Y}, \tilde{F}_{Z}$ : aerodynamic forces caused by the right or left flapping wing in the $X, Y,-Z$ directions

$\tilde{F}_{X P}, \tilde{F}_{Y P}, \tilde{F}_{Z P}$ : aerodynamic forces caused by one propeller in the $X, Y,-Z$ directions

$F_{Y P}(t)$ : time-variation of $F_{Y P}$, generated by one blade of one propeller

$f$ : flapping frequency

$\bar{f}$ : flapping frequency with no wind gust $h$ : distance of origins between $X_{B}-Y_{B}-Z_{B}$ and direction $X-Y-Z$ in the $X$ direction

$\tilde{P}$ : power consumed by the right or left flapping wing

$\bar{P}$ : power consumed by the right and left flapping wings

$\tilde{P}_{P}$ : power consumed by one propeller

$\bar{P}_{P}$ : power consumed by all propellers

$R$ : flapping wing length or half span length of the fixed wing or propeller radius

$R_{P}$ : propeller radius

$r$ : spanwise position

T: period

$t$ : time

$U_{T}$ : air velocity of wing section, tangential to the disc plane

$U_{P}$ : air velocity of wing section, perpendicular to the disc plane

$V$ : forward velocity

$v_{i}$ : induced velocity of an ornithopter in the $Z$ direction

$v_{i X}, v_{i Y}, v_{i Z}$ : induced velocity of a propeller in the $X, Y$, $Z$ directions

$w_{X}, w_{Y}, w_{Z}$ : wind gusts in the $-X,-Y,-Z$ directions

$X-Y-Z$ : body fixed coordinate system

$\alpha$ : angle of attack

$\beta$ : torsional angle

$\Delta F_{\text {air }}$ : difference of aerodynamic force acting on an airplane with and without wind gusts

$\Delta F_{\text {orn }}$ : difference of aerodynamic force acting on an ornithopter with and without wind gusts

$\delta$ : drag coefficient

$\phi$ : flapping angle or rotational angle

$\phi_{1}$ : amplitude of flapping angle

$\theta$ : feathering angle

$\theta_{0}$ : mean feathering angle 
$\theta_{1}$ : amplitude of feathering angle

$\rho$ : air density

$\Omega$ : rotational speed

$\bar{\Omega}$ : rotational speed with no wind gust

$\xi$ : angle between inflow velocity and disc plane

\section{Introduction}

Recently, small and lightweight airplanes, such as micro air vehicles (MAVs) have been developed. However, these airplanes have a serious problem: they are strongly affected by wind gusts. However, birds and insects seem to fly stably in wind, even though they are smaller and more lightweight than MAVs. Several stability analyses on insect flight have revealed that it is inherently unstable. During flight, an insect stabilizes its body's motion by active control using information from its eyes and halters. ${ }^{1-7)}$ In a wind gust, insects and birds may control their flight better than MAVs.

The big difference between airplanes and birds/insects is that the former generate thrust by propellers and the latter by flapping their wings. This difference might cause the difference in the robustness of flight in a wind gust. Sigthorsson et al. ${ }^{8)}$ investigated a similar problem between a flapping wing vehicle and a rotorcraft. They showed that a flapping wing vehicle is generally less sensitive to velocity perturbations in all aerodynamic forces and moments than a rotorcraft, except for a forward/backward-direction forces. In the present paper, the variation in aerodynamic forces acting on an ornithopter MAV and that on a fixed-wing MAV with propeller(s) is compared under wind gust conditions in order to investigate how the former is less sensitive to a wind gust. ${ }^{9)}$ The variation in aerodynamic forces in the analysis corresponds to stability derivatives concerning variation in angle of attack, side slip angle and forward velocity. It is assumed that smaller values of these stability derivatives are required for a better aircraft. Note that aircraft in the analysis have sufficient flight control ability.

\section{Analysis}

The variation in aerodynamic forces acting on an airplane with one or two propellers when it encounters a wind gust is compared. The size of the beating wing of the ornithopter, the main wing and the propeller blade of the airplane, and the motions of the beating wing and the propeller are parameters. These are determined as follows: Shape and size of the main wing of the airplane are the same as those of the beating wings of the ornithopter. In addition, their size, which is assumed in the present analysis, is close to that of a small unmanned aerial vehicle (UAV). When they do not encounter a wind gust, the following points are satisfied. The two kinds of flapping motions of the beating wings are assumed. The feathering motion of the beating wings is close to that of a bird. The parasite drag on the fuselage and the total mass of the ornithopter are determined so that they are equal to the thrust (horizontal force) and the lift (vertical force) by the beating wings, respectively. They are equal to the parasite drag on the fuselage of the airplane and the total mass of the airplane, respectively. The time-averaged flapping and feathering angles of the ornithopter are equal to the dihedral angle and pitch angle of the main wing of the airplane, respectively. The airplane has one or two propellers. The number of blades of each propeller is four. Some kinds of propeller(s), which have various diameters and chord lengths, are assumed. The resultant angle of attack of each blade elements is $5^{\circ}$, where the lift-drag ratio is high. The rotational speed of propellers is varied so that the thrust generated by the propellers is equal to the parasite drag on the fuselage. Note that the thrust generated by the propellers is equal to that generated by the beating wings. The present analysis is based on blade element theory and momentum theory and the quasi-steady assumption is applied.

In the analysis, the vertical and horizontal tails of the airplane are ignored. The interference among the fuselage, the propellers and the main wing of the airplane and that between the fuselage and the beating wings of the ornithopter are ignored.

\subsection{Ornithopter}

An ornithopter with two flapping wings of wing length $R$, chord length $c$ and flapping frequency $f$ encounters winds in the $-X,-Y$ and $-Z$ directions, $w_{X}, w_{Y}$ and $w_{Z}$. The stroke plane of the flapping wings is vertical. Flapping angle $\phi$ and feathering angle $\theta$ are given by

$$
\phi=\phi_{1} \cos (2 \pi f t), \quad \theta=\theta_{0}+\theta_{1} \sin (2 \pi f t) .
$$

The phase difference between the flapping and feathering angles is $90^{\circ}$, where the flight efficiency is maximum. ${ }^{10)}$ As shown in Fig. 1, the lift and drag acting on a wing element at $r$ are

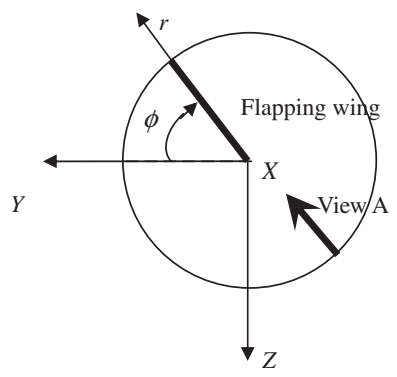

(a)

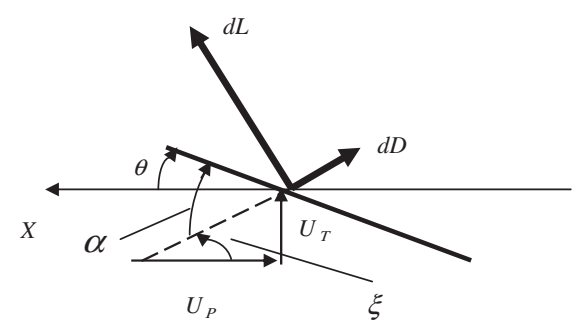

(b)

Fig. 1. Lift and drag acting on the flapping wing element: (a) flapping angle $\phi$ and (b) view A. 
$\mathrm{d} L=0.5 \rho\left(U_{T}^{2}+U_{P}^{2}\right) C_{\ell \alpha} \alpha c \mathrm{~d} r, \quad \mathrm{~d} D=0.5 \rho\left(U_{T}^{2}+U_{P}^{2}\right) \delta c \mathrm{~d} r$,

where $\quad U_{T}=-r \dot{\phi}+\left(w_{Z}-v_{i}\right) \cos \phi \pm w_{Y} \sin \phi, \quad U_{P}=$ $V+w_{X}, \alpha=\theta+\xi$ and $\tan \xi=U_{T} / U_{P}$. In this section, the upper and lower signs denote the right and left wings, and $v_{i}$ is the induced velocity in the $Z$ direction and is given by

$$
v_{i}=\frac{F_{Z}}{2 \pi \rho R^{2} V},
$$

where $F_{Z}$ is an aerodynamic force in the $-Z$ direction, which is defined later. The induced velocities in $X$ and $Y$ are ignored. The aerodynamic forces generated by a right or left wing in the $X, Y$ and $-Z$ directions, their aerodynamic moments around the $X, Y$ and $Z$ axes, and their consumed power are given by

$$
\begin{gathered}
\tilde{F}_{X}=-\frac{1}{T} \int_{0}^{T} \int_{0}^{R}(\mathrm{~d} L \sin \xi+\mathrm{d} D \cos \xi) \mathrm{d} t \\
\tilde{F}_{Y}=\mp \frac{1}{T} \int_{0}^{T} \int_{0}^{R}(\mathrm{~d} L \cos \xi-\mathrm{d} D \sin \xi) \sin \phi \mathrm{d} t \\
\tilde{F}_{Z}=\frac{1}{T} \int_{0}^{T} \int_{0}^{R}(\mathrm{~d} L \cos \xi+\mathrm{d} D \sin \xi) \cos \phi \mathrm{d} t \\
\tilde{P}=-\frac{1}{T} \int_{0}^{T} \int_{0}^{R} r(\mathrm{~d} L \cos \xi+\mathrm{d} D \sin \xi) \dot{\phi} \mathrm{d} t
\end{gathered}
$$

The lift and drag coefficients of a two-dimensional wing are assumed to be $C_{\ell}=5.73 \alpha$ and $\delta=0.02$.

The horizontal force generated by the right and left wings at $w_{X}=w_{Y}=w_{Z}=0, \bar{F}_{X}$, is equal to the parasite drag acting on body $\bar{D}_{F}$ when the ornithopter makes a trimmed flight.

\subsection{Airplane}

\subsubsection{Main wing}

When $f=0$ and $\theta_{1}=0$, the ornithopter analysis focuses on an airplane's main fixed wing with a span of $2 R$.

\subsubsection{Propeller}

The airplane has one or two propellers. A propeller has four blades $(b=4)$, and their radii, chords and rotational speeds are $R_{P}, c_{P}$ and $\Omega=\dot{\phi}$, respectively. The horizontal force generated by all the propellers at $w_{X}=w_{Y}=w_{Z}=$ $0, \bar{F}_{X P}$, is given by

$$
\bar{F}_{X P}=-\bar{F}_{X W}+\bar{D}_{F}
$$

The following are the induced velocities in the $X, Y$ and $Z$ directions by one propeller.

$v_{i X}=\frac{-\tilde{F}_{X P}}{2 \pi \rho R^{2} V}, \quad v_{i Y}=\frac{-\tilde{F}_{Y P}}{2 \pi \rho R^{2} V}, \quad v_{i Z}=\frac{\tilde{F}_{Z P}}{2 \pi \rho R^{2} V}$

As shown in Fig. 2, the lift and drag acting on a wing element at $r$ are

$$
\begin{aligned}
\mathrm{d} L & =0.5 \rho\left(U_{T}^{2}+U_{P}^{2}\right) C_{\ell \alpha} \alpha c_{P} \mathrm{~d} r, \\
\mathrm{~d} D & =0.5 \rho\left(U_{T}^{2}+U_{P}^{2}\right) \delta c_{P} \mathrm{~d} r,
\end{aligned}
$$

where $U_{P}=V-v_{i X}+w_{X}, U_{T}=r \Omega+\left(-v_{i Z}+w_{Z}\right) \sin \phi \pm$ $\left(-v_{i Y}+w_{Y}\right) \cos \phi, \alpha=\beta+\xi-\pi / 2$ and $\tan \xi=U_{T} / U_{P}$. In this section, the upper and lower signs are for a propeller

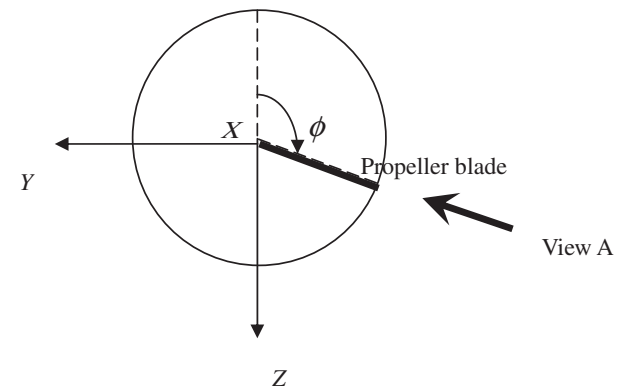

(a)

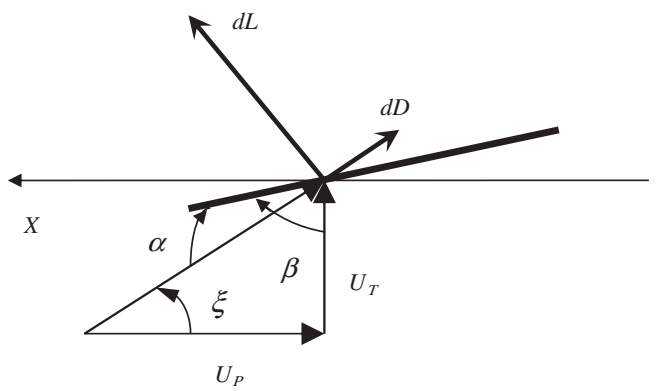

(b)

Fig. 2. Lift and drag acting on the propeller element: (a) rotational angle $\phi$ and (b) view A.

rotating clockwise and counterclockwise when viewed along the $X$ direction. The aerodynamic forces by one propeller in the $X, Y$ and $Z$ directions, their aerodynamic moments around the $X, Y$ and $Z$ axes, and their consumed power are given by

$$
\begin{aligned}
\tilde{F}_{X P} & =\frac{b}{T} \int_{0}^{T} \int_{0}^{R}(\mathrm{~d} L \sin \xi-\mathrm{d} D \cos \xi) \mathrm{d} t \\
\tilde{F}_{Y P} & = \pm \frac{b}{T} \int_{0}^{T} \cos \phi \int_{0}^{R}(\mathrm{~d} L \cos \xi-\mathrm{d} D \sin \xi) \mathrm{d} t \\
\tilde{F}_{Z P} & =\frac{b}{T} \int_{0}^{T} \sin \phi \int_{0}^{R}(\mathrm{~d} L \cos \xi+\mathrm{d} D \sin \xi) \mathrm{d} t \\
\tilde{P}_{P} & =\frac{b \Omega}{T} \int_{0}^{T} \int_{0}^{R} r(\mathrm{~d} L \cos \xi+\mathrm{d} D \sin \xi) \mathrm{d} t .
\end{aligned}
$$

The torsional angle is given by

$$
\beta(r)=5^{\circ}+\tan ^{-1}\left(\frac{V+v_{i X}}{r \Omega}\right) .
$$

The angle of attack at any blade element is $5^{\circ}$ without wind gusts. The lift and drag coefficients of a two-dimensional wing are assumed to be $C_{\ell}=5.73 \alpha$ and $\delta=0.02$, which are similar to those of the ornithopter. In the present analysis, the tail wings of the airplane and interference between the main wing and propellers are ignored.

\section{Results}

\subsection{Forces}

\subsubsection{Ornithopter}

Table 1 shows the horizontal and vertical forces generated by the ornithopter, $\bar{F}_{X}$ and $\bar{F}_{Z}$, and their consumed 
Table 1. Aerodynamic force generated by the ornithopter with no wind gust.

\begin{tabular}{cccccc}
\hline Case & $\bar{f}$ & $\bar{F}_{Z}$ & $\bar{F}_{X}$ & $\begin{array}{c}\bar{D}_{F} \\
(\mathrm{~N})\end{array}$ & $\begin{array}{c}\bar{P} \\
(\mathrm{~W})\end{array}$ \\
\hline A & 4.77 & 2.59 & 0.332 & 0.332 & 9.85 \\
B & 1.59 & 2.48 & 0.171 & 0.171 & 6.56 \\
\hline
\end{tabular}

Table 3. Aerodynamic force generated by the airplane with no wind gust.

\begin{tabular}{cccccccc}
\hline \multirow{2}{*}{ Case } & \multicolumn{2}{c}{ Fixed wing } & & Fuselage & & \multicolumn{2}{c}{ Propeller } \\
\cline { 2 - 3 } \cline { 7 - 8 } \cline { 7 - 8 } & $\bar{F}_{Z W}$ & $\bar{F}_{X W}$ & & $\bar{D}_{F}$ & & $\bar{F}_{X P}$ & $\bar{F}_{Z P}$ \\
& $(\mathrm{~N})$ & $(\mathrm{N})$ & & $(\mathrm{N})$ & & $(\mathrm{N})$ & $(\mathrm{N})$ \\
\hline A & 2.59 & -0.157 & & 0.332 & & 0.489 & 0 \\
B & 2.59 & -0.157 & & 0.171 & & 0.328 & 0 \\
\hline
\end{tabular}

Table 2. Aerodynamic force generated by the ornithopter encountering wind gust.

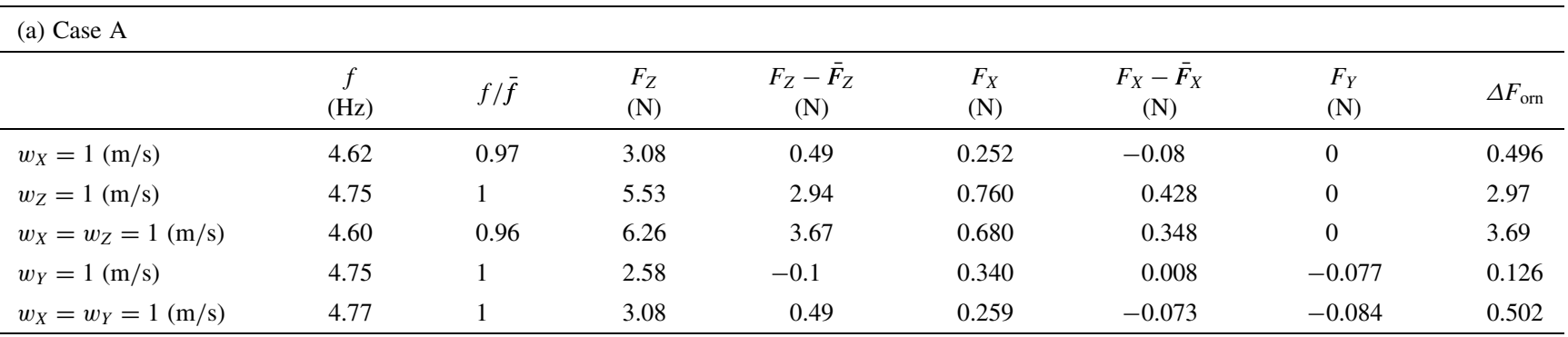

(b) Case B

\begin{tabular}{|c|c|c|c|c|c|c|c|c|}
\hline & $\begin{array}{c}f \\
(\mathrm{~Hz})\end{array}$ & $f / \bar{f}$ & $\begin{array}{l}F_{Z} \\
(\mathrm{~N})\end{array}$ & $\begin{array}{c}F_{Z}-\bar{F}_{Z} \\
(\mathrm{~N})\end{array}$ & $\begin{array}{l}F_{X} \\
(\mathrm{~N})\end{array}$ & $\begin{array}{c}F_{X}-\bar{F}_{X} \\
(\mathrm{~N})\end{array}$ & $\begin{array}{l}F_{Y} \\
(\mathrm{~N})\end{array}$ & $\Delta F_{\text {orn }}$ \\
\hline$w_{X}=1(\mathrm{~m} / \mathrm{s})$ & 1.54 & 0.97 & 2.95 & 0.47 & 0.107 & -0.064 & 0 & 0.474 \\
\hline$w_{Z}=1(\mathrm{~m} / \mathrm{s})$ & 1.58 & 0.99 & 5.14 & 2.66 & 0.579 & 0.408 & 0 & 2.69 \\
\hline$w_{X}=w_{Z}=1(\mathrm{~m} / \mathrm{s})$ & 1.54 & 0.97 & 5.84 & 3.36 & 0.517 & 0.346 & 0 & 3.38 \\
\hline$w_{Y}=1(\mathrm{~m} / \mathrm{s})$ & 1.59 & 1 & 2.48 & 0 & 0.216 & 0.045 & -0.451 & 0.453 \\
\hline$w_{X}=w_{Y}=1(\mathrm{~m} / \mathrm{s})$ & 1.54 & 0.97 & 2.95 & 0.47 & 0.152 & -0.019 & -0.496 & 0.684 \\
\hline
\end{tabular}

power $\bar{P}$ for cases A and B. In both cases, $R=0.5 \mathrm{~m}$, $c=0.1 \mathrm{~m}, \theta_{0}=5^{\circ}, \theta_{1}=2^{\circ}, V=10 \mathrm{~m} / \mathrm{s}$ and $w_{X}=w_{Y}=$ $w_{Z}=0$. The following are different for cases $\mathrm{A}$ and $\mathrm{B}$ :

Case A: $\bar{f}=30 / 2 \pi(1 / \mathrm{s}), \quad \phi_{1}=12^{\circ}$.

Case B: $\bar{f}=10 / 2 \pi(1 / \mathrm{s}), \quad \phi_{1}=30^{\circ}$.

Parasite drag on body $\bar{D}_{F}$ is determined to equal horizontal force $\bar{F}_{X}$. Table 2 shows $F_{X}, F_{Y}$ and $F_{Z}$ when the ornithopter encounters wind gusts: $w_{X}=1 \mathrm{~m} / \mathrm{s}, w_{Z}=1 \mathrm{~m} / \mathrm{s}$, $w_{Y}=1 \mathrm{~m} / \mathrm{s}, \quad w_{X}=w_{Z}=1 \mathrm{~m} / \mathrm{s}$ and $w_{X}=w_{Y}=1 \mathrm{~m} / \mathrm{s}$. Except for the flapping frequency when the ornithopter encounters a wind gust, the wing motion is the same as that with no wind gust. The consumed power when the ornithopter encounters a wind gust is the same as that with no wind gust. The unsteady effect on the aerodynamic forces when the ornithopter encounters a wind gust is ignored. The differences of the aerodynamic forces acting on the ornithopter under wind gusts from those under no wing gust are given by

$$
\Delta F_{\text {orn }}=\left[\left(F_{Z}-\bar{F}_{Z}\right)^{2}+\left(F_{X}-\bar{F}_{X}\right)^{2}+F_{Y}^{2}\right]^{0.5} .
$$

Here, the variation of the parasite drag on the fuselage is ignored. Comparing $\Delta F_{\text {orn }}$ between cases $\mathrm{A}$ and $\mathrm{B}$ in Table 2, a big difference is observed when $w_{Y}=1 \mathrm{~m} / \mathrm{s}$. The $\Delta F_{\text {orn }}$ for case $\mathrm{A}$ is much smaller than that for case B. This can be explained by the following two reasons: (1) The wing speed, which is proportional to $\phi_{1} f$, of case $\mathrm{A}$ is larger than that of case $\mathrm{B}$. The ratio of a wind gust to the wing speed of case A is smaller than that of case B. (2) Furthermore, the projected area of a wing sweeping region to the $X-Z$ plane of case $\mathrm{A}$ is smaller than that of case B. Note that $w_{Y}$ is perpendicular to the $X-Z$ plane.

\subsubsection{Airplane}

Table 3 shows the vertical and horizontal forces generated by a fixed wing of an airplane, $\bar{F}_{Z W}$ and $\bar{F}_{X W}$, when the values of $R=0.5 \mathrm{~m}, c=0.1 \mathrm{~m}, \theta_{0}=5^{\circ}, V=10 \mathrm{~m} / \mathrm{s}$ and $w_{X}=w_{Y}=w_{Z}=0$ are given. The fixed wing has no torsion along the span. $\bar{F}_{Z W}$ equals the $\bar{F}_{Z}$ of the ornithopter for case A. For case B, the airplane's $\bar{F}_{Z W}$ is slightly larger than $\bar{F}_{Z}$. This small difference between the ornithopter and the airplane with no wind gust is ignored when the aerodynamic forces on them with wind gusts are compared. $\bar{D}_{F}$ on the airplane's fuselage is identical to that on the ornithopter's fuselage. The $\bar{F}_{X W}$ is calculated using the simple momentum and blade element theories. Total propeller thrust $\bar{F}_{X P}$ is determined via Eq. (5).

For cases A and B, six and seven kinds of propellers are mounted on the airplane, respectively. Cases A1-6 and B17 , which generate $\bar{F}_{X P}$, are considered. The airplane has one propeller in cases A1-2 and B1-3 and two in cases A3-6 and B4-7. In cases A1-2 and B1-3, the propeller rotates counterclockwise when viewed along the $X$ direction. In cases A3-6 and B4-7, one propeller rotates clockwise and the other counterclockwise.

The dimensions of $R_{P}, c_{P}, \bar{\Omega}$ and $\bar{P}_{P}$ are shown in Table 4 . Note that $\bar{P}_{P}$ is the power consumed by the two propellers in cases A3-6 and B4-7.

The aerodynamic forces on the main wing when the airplane encounters the gusts are shown in Table 5. The aero- 
Table 4. Dimensions of propeller(s) of the airplane and power consumed by propeller(s) with no wind gust.

(a) Case A1-6

\begin{tabular}{ccccccc}
\hline Case & $\begin{array}{c}R_{P} \\
(\mathrm{~m})\end{array}$ & $\begin{array}{c}c_{P} \\
(\mathrm{~m})\end{array}$ & $R_{P} / c_{P}$ & $\begin{array}{c}\bar{\Omega} \\
(\mathrm{rad} / \mathrm{s})\end{array}$ & $\begin{array}{c}\bar{P}_{P} \\
(\mathrm{~W})\end{array}$ & $R_{P} \bar{\Omega} / V$ \\
\hline $\mathrm{A} 1$ & 0.5 & 0.1 & 5 & 4.8 & 7.50 & 0.24 \\
$\mathrm{~A} 2$ & 0.2 & 0.04 & 5 & 46 & 5.61 & 0.92 \\
$\mathrm{~A} 3$ & 0.2 & 0.05 & 4 & 23 & 6.06 & 0.46 \\
$\mathrm{~A} 4$ & 0.2 & 0.035 & 5.7 & 30 & 5.73 & 0.6 \\
$\mathrm{~A} 5$ & 0.1 & 0.02 & 5 & 146 & 5.57 & 1.46 \\
$\mathrm{~A} 6$ & 0.1 & 0.01 & 10 & 225 & 5.57 & 2.25 \\
\hline
\end{tabular}

(b) Case B1-7

\begin{tabular}{lllcccc}
\hline Case & $\begin{array}{c}R_{P} \\
(\mathrm{~m})\end{array}$ & $\begin{array}{c}c_{P} \\
(\mathrm{~m})\end{array}$ & $R_{P} / c_{P}$ & $\begin{array}{c}\bar{\Omega} \\
(\mathrm{rad} / \mathrm{s})\end{array}$ & $\begin{array}{c}\bar{P}_{P} \\
(\mathrm{~W})\end{array}$ & $R_{P} \bar{\Omega} / V$ \\
\hline B1 & 0.5 & 0.1 & 5 & 3.7 & 5.73 & 0.19 \\
B2 & 0.5 & 0.024 & 21 & 10 & 3.94 & 0.5 \\
B3 & 0.2 & 0.04 & 5 & 34 & 3.81 & 0.68 \\
B4 & 0.2 & 0.05 & 4 & 17 & 4.34 & 0.34 \\
B5 & 0.2 & 0.11 & 1.8 & 10 & 5.50 & 0.2 \\
B6 & 0.1 & 0.02 & 5 & 112 & 3.69 & 1.12 \\
B7 & 0.1 & 0.01 & 10 & 177 & 3.68 & 1.77 \\
\hline
\end{tabular}

Table 5. Aerodynamic force generated by the airplane's fixed wing encountering wind gust.

\begin{tabular}{lccccc}
\hline & $\begin{array}{c}F_{Z W} \\
(\mathrm{~N})\end{array}$ & $\begin{array}{c}F_{X W} \\
(\mathrm{~N})\end{array}$ & $\begin{array}{c}F_{Y W} \\
(\mathrm{~N})\end{array}$ & $\begin{array}{c}F_{Z W}-\bar{F}_{Z W} \\
(\mathrm{~N})\end{array}$ & $\begin{array}{c}F_{X W}-\bar{F}_{X W} \\
(\mathrm{~N})\end{array}$ \\
\hline $\begin{array}{l}w_{X}=1 \\
(\mathrm{~m} / \mathrm{s})\end{array}$ & 3.09 & -0.193 & 0 & 0.5 & -0.036 \\
$\begin{array}{l}w_{Z}=1 \\
(\mathrm{~m} / \mathrm{s})\end{array}$ & 5.57 & 0.273 & 0 & 2.98 & 0.43 \\
$\begin{array}{l}w_{X}=w_{Z}=1 \\
(\mathrm{~m} / \mathrm{s})\end{array}$ & 6.32 & 0.237 & 0 & 3.73 & 0.394 \\
$\begin{array}{l}w_{Y}=1 \\
(\mathrm{~m} / \mathrm{s})\end{array}$ & 2.59 & -0.157 & 0 & 0 & 0 \\
$\begin{array}{l}w_{X}=w_{Y}=1 \\
(\mathrm{~m} / \mathrm{s})\end{array}$ & 3.09 & -0.193 & 0 & 0.5 & -0.036 \\
\hline
\end{tabular}

dynamic forces at $w_{Y}=1 \mathrm{~m} / \mathrm{s}$ are assumed to be zero because the dihedral angle of the fixed wing is zero.

The difference in aerodynamic forces acting on the airplane with and without wind gust, is given by

$\Delta F_{\text {air }}=\left[\left(F_{Z P}+\Delta F_{Z W}\right)^{2}+\left(\Delta F_{X P}+\Delta F_{X W}\right)^{2}+F_{Y P}{ }^{2}\right]^{0.5}$,

where $\Delta F_{Z W}=F_{Z W}-\bar{F}_{Z W}, \Delta F_{X P}=F_{X P}-\bar{F}_{X P}, \Delta F_{X W}=$ $F_{X W}-\bar{F}_{X W}$.

Here, the variation of parasite drag on the fuselage is ignored. The $\beta$ of the propeller(s) when the airplane encounters a wind gust is the same as that with no wind gust. The rotational speed of the propellers is varied, and the consumed power when the airplane encounters a wind gust is identical to that with no wind gust. The unsteady effect on the aerodynamic forces is ignored when the airplane encounters a wind gust.
Figures 3 and 4 show the values of terms in Eq. (11) for cases A and B, respectively. Figures 3 and 4(a), (b), (c), (d) and (e) show the values when $w_{X}=1 \mathrm{~m} / \mathrm{s}, w_{Z}=1 \mathrm{~m} / \mathrm{s}$, $w_{Y}=1 \mathrm{~m} / \mathrm{s}, \quad w_{X}=w_{Z}=1 \mathrm{~m} / \mathrm{s}$ and $w_{X}=w_{Y}=1 \mathrm{~m} / \mathrm{s}$, respectively. Figures 3(a) and 4(a) show that the variation in aerodynamic forces acting on a fixed wing in the $Z$ direction, $\Delta F_{Z W}$, is dominant when $w_{X}=1 \mathrm{~m} / \mathrm{s}$. Note that the direction of $\Delta F_{Z W}$ is perpendicular to the wind gust. Figures 3(b) and 4(b) show that the variation in aerodynamic forces acting on a fixed wing in the $Z$ direction, $\Delta F_{Z W}$, is dominant when $w_{Z}=1 \mathrm{~m} / \mathrm{s}$, independently of $R_{P} \bar{\Omega} / V$. Furthermore, when $R_{P} \bar{\Omega} / V$ is small, the variation in aerodynamic forces acting on propellers in the $Z$ direction, $F_{Z P}$, is also large. Figures 3(c) and 4(c) show that, when $w_{Y}=1 \mathrm{~m} / \mathrm{s}$, the variation of aerodynamic force on propellers in the $Y$ direction, $F_{Y P}$, is not negligible at small $R_{P} \bar{\Omega} / V$. Figures $3(\mathrm{~d})$ and $4(\mathrm{~d})$, where $w_{X}=w_{Z}=1 \mathrm{~m} / \mathrm{s}$, can be obtained by superposing Figs. 3(a) and 4(a) $\left(w_{X}=1 \mathrm{~m} / \mathrm{s}\right)$ and $3(\mathrm{~b})$ and $4(\mathrm{~b})\left(w_{Z}=1 \mathrm{~m} / \mathrm{s}\right)$, respectively. Similarly, Figures 3(e) and 4(e), where $w_{X}=w_{Y}=1 \mathrm{~m} / \mathrm{s}$, can be obtained by superposing Figs. 3(a) and 4(a) $\left(w_{X}=1 \mathrm{~m} / \mathrm{s}\right)$ and $3(\mathrm{c})$ and $4(\mathrm{c})\left(w_{Y}=1 \mathrm{~m} / \mathrm{s}\right)$, respectively.

Figures 5(a) and (b) show the values of $\Delta F_{\text {air }} / \Delta F_{\text {orn }}$ for the comparisons between case $\mathrm{A}$ (ornithopter) and case A1-6 (airplane) and those between case B (ornithopter) and case B1-7 (airplane), respectively. In these figures, the values of the horizontal axes are $R_{P} \bar{\Omega} / V$, and the values of $\Delta F_{\text {air }} / \Delta F_{\text {orn }}$ when $w_{X}=1 \mathrm{~m} / \mathrm{s}, w_{Z}=1 \mathrm{~m} / \mathrm{s}, w_{Y}=1 \mathrm{~m} / \mathrm{s}$, $w_{X}=w_{Z}=1 \mathrm{~m} / \mathrm{s}$ and $w_{X}=w_{Y}=1 \mathrm{~m} / \mathrm{s}$ are shown. The values of $\bar{P}_{P} / \bar{P}$ are also shown in these figures. The values of $\bar{P}_{P} / \bar{P}$ are less than one. Power consumed by the flapping wings is larger than that consumed by the propellers. The $\Delta F_{\text {air }} / \Delta F_{\text {orn }}$ decreases with the increase of $R_{P} \bar{\Omega} / V$. This is because the $\Delta F_{\text {air }}$ calculated using the terms in Eq. (11) decreases with the increase of $R_{P} \bar{\Omega} / V$. The $\Delta F_{\text {air }} / \Delta F_{\text {orn }}$ are larger than one except for when $w_{Y}=1 \mathrm{~m} / \mathrm{s}$ in Figs. 5(a) and (b) and $w_{X}=w_{Y}=1 \mathrm{~m} / \mathrm{s}$ in Fig. 5(b). The $\Delta F_{\text {air }} / \Delta F_{\text {orn }}$ for $w_{Y}=1 \mathrm{~m} / \mathrm{s}$ in Figs. 5(a) and (b) and $w_{X}=w_{Y}=1 \mathrm{~m} / \mathrm{s}$ in Fig. 5(b) are larger than one when $R_{P} \bar{\Omega} / V$ is small. On the other hand, the $\Delta F_{\text {air }} / \Delta F_{\text {orn }}$ for these cases are smaller than one when $R_{P} \bar{\Omega} / V$ is large. When $R_{P} \bar{\Omega} / V$ is smaller than 2.1 and 0.7 for cases $\mathrm{A}$ and $\mathrm{B}$, respectively, the $\Delta F_{\text {air }} / \Delta F_{\text {orn }}$ for all the wind gusts are larger than one. At these values of $R_{P} \bar{\Omega} / V$, the ornithopter is affected more weakly than the airplane with the propellers for all the wind gusts. The criteria of $R_{P} \bar{\Omega} / V$ in case $\mathrm{A}$ (2.1) is larger than that in case B (0.7). The ornithopter in case A is more weakly affected by a side wind gust than that in case B. It can be expected that the effect of a side wind gust decreases when the flapping velocity increases and the flapping amplitude decreases. Since the flapping velocities in cases A and B are similar, the smaller flapping amplitude in case A mainly contributes to a weaker effect of a side wind gust. As stated above, when $R_{P} \bar{\Omega} / V$ of a propeller and $\phi_{1}$ of an ornithopter are smaller, the $\Delta F_{\text {air }} / \Delta F_{\text {orn }}$ is larger and the ornithopter is affected more weakly by a side wind gust than the airplane. 


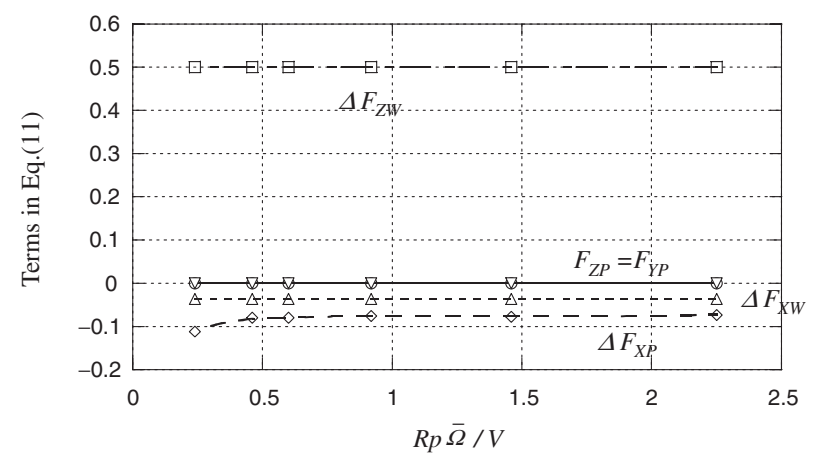

(a)

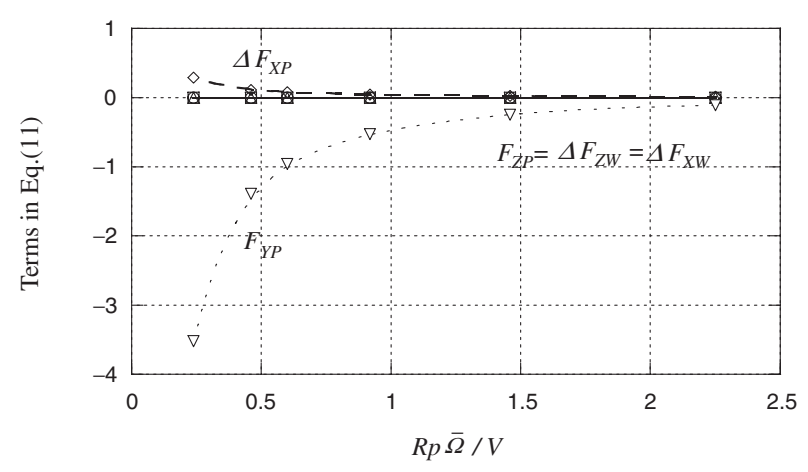

(c)

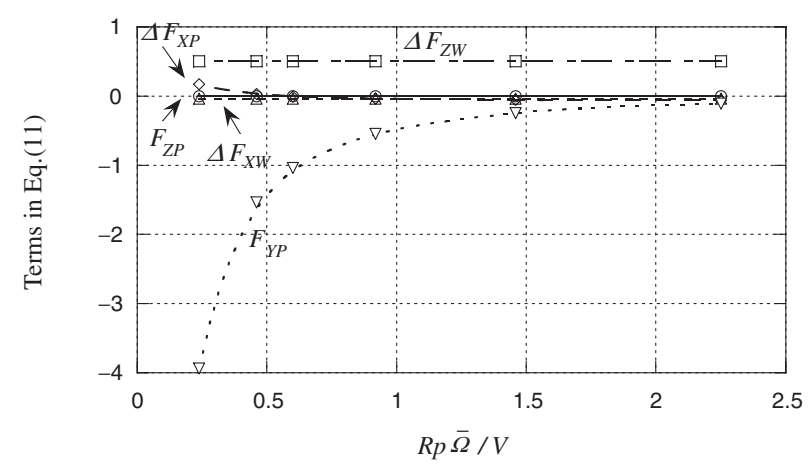

(e)

When the ornithopter encounters all the wind gusts, the variations of flapping frequency are very small as indicated in Table 2. When the airplane encounters the wind gusts $w_{Z}=1 \mathrm{~m} / \mathrm{s}, w_{Y}=1 \mathrm{~m} / \mathrm{s}$ and $w_{Y}=w_{Z}=1 \mathrm{~m} / \mathrm{s}$, the rotational speed of the propellers does not vary largely. On the other hand, when it encounters the wind gusts $w_{X}=1$, $w_{X}=w_{Z}=1 \mathrm{~m} / \mathrm{s}$ and $w_{X}=w_{Y}=1 \mathrm{~m} / \mathrm{s}$, wind gusts with a component in the $X$ direction, the rotational speed of the propellers varies largely. The variation in aerodynamics forces when consumed power is fixed are compared for the ornithopter and the airplane with propellers in the present analysis. Furthermore, the flapping frequencies of the ornithopter and the rotational speed of the propellers are nearly constant when they encounter the wind gusts $w_{Z}=$ $1 \mathrm{~m} / \mathrm{s}, w_{Y}=1 \mathrm{~m} / \mathrm{s}$ and $w_{Y}=w_{Z}=1 \mathrm{~m} / \mathrm{s}$.

Figure 6 shows the variation in rotational speed of the propellers in cases $\mathrm{A} 1-6$ and $\mathrm{B} 1-7, \Omega / \bar{\Omega}$, when the airplane encounters the wind gusts $w_{X}=1 \mathrm{~m} / \mathrm{s}$. The values shown in this figure are nearly the same as those when they encounter

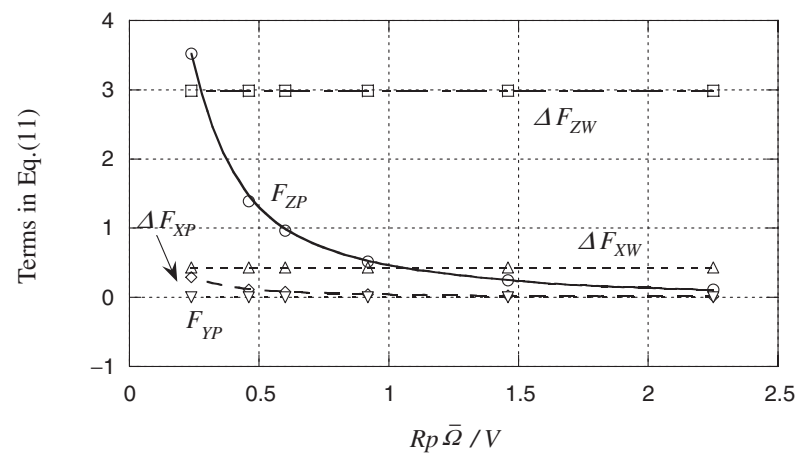

(b)

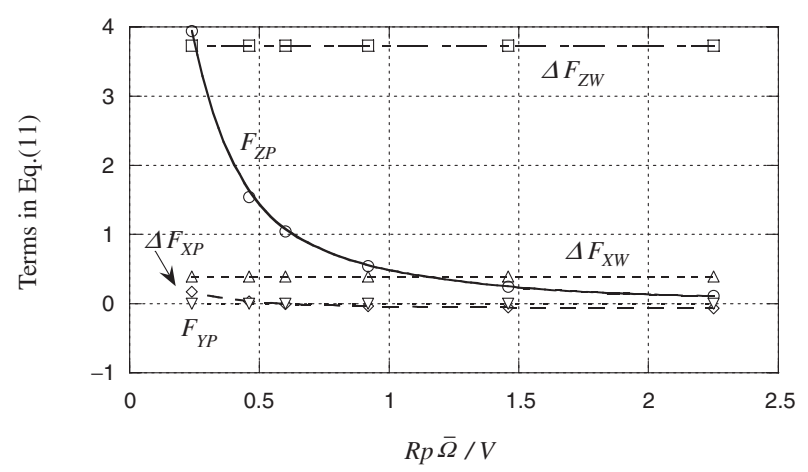

(d)

Fig. 3. Values of the terms in Eq. (11) for case A: (a) $w_{X}=1 \mathrm{~m} / \mathrm{s}$, (b) $w_{Z}=1 \mathrm{~m} / \mathrm{s}$, (c) $w_{Y}=1 \mathrm{~m} / \mathrm{s}$, (d) $w_{X}=w_{Z}=1 \mathrm{~m} / \mathrm{s}$ and (e) $w_{X}=w_{Y}=1 \mathrm{~m} / \mathrm{s}$.

the wind gusts $w_{X}=w_{Y}=1 \mathrm{~m} / \mathrm{s}$ and $w_{X}=w_{Z}=1 \mathrm{~m} / \mathrm{s}$. The points for case A1-6 and those for case B1-7 are nearly on one line in this figure. Then, the values for case A1-6 agree with those for case B1-7 when the $R_{P} \bar{\Omega} / V$ is common. The $\Omega / \bar{\Omega}$ depends solely on the $R_{P} \bar{\Omega} / V$ and they are larger than one when $R_{P} \bar{\Omega} / V>0.3$.

Figure 7 shows the time-variation of $F_{Y P}(t)$ for $w_{Y}=1 \mathrm{~m} / \mathrm{s}$, which is generated by one blade of one propeller in case A4. Note that Fig. 5(a) shows that the $\Delta F_{\text {air }} / \Delta F_{\text {orn }}$ for $w_{Y}=1 \mathrm{~m} / \mathrm{s}$ at $R_{P} \bar{\Omega} / V=0.6$ (case A4) is much larger than one. This propeller rotates counterclockwise when viewed along the $X$ direction. A large difference of $F_{Y P}(t)$ can be observed around $\phi \approx \pi$ and around $\phi \approx 0$. This difference causes $F_{Y P}$, which is a time-averaged $F_{Y P}(t)$ by the two propellers. The difference of $F_{Y P}(t)$ around $\phi \approx \pi$ and around $\phi \approx 0$ is explained as follows. (1) Figure 8 shows the variation in the angle of attack of the propeller at a $75 \%$ spanwise position for case A4. The variation is also shown when $w_{X}=1 \mathrm{~m} / \mathrm{s}$, 


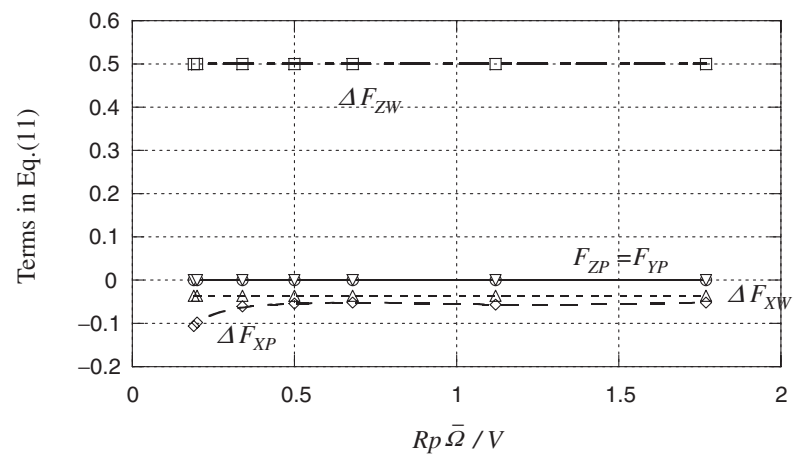

(a)

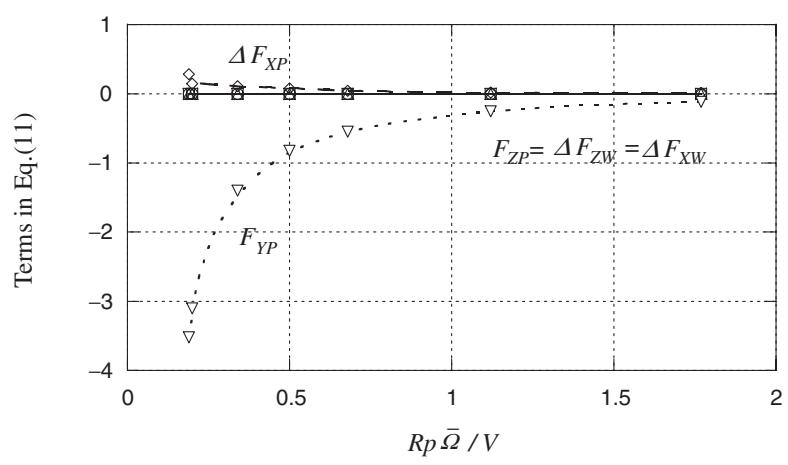

(c)

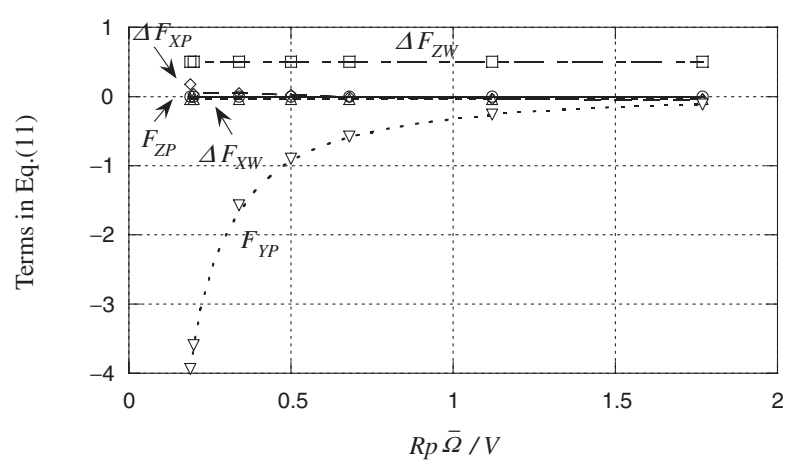

(e)

$w_{Z}=1 \mathrm{~m} / \mathrm{s}, \quad w_{Y}=1 \mathrm{~m} / \mathrm{s}$, and $w_{X}=w_{Y}=w_{Z}=0 \mathrm{~m} / \mathrm{s}$. When $w_{Y}=1 \mathrm{~m} / \mathrm{s}$ and $w_{Z}=1 \mathrm{~m} / \mathrm{s}, \alpha$ is very different from that with no wind gust. The difference when $w_{Y}=1 \mathrm{~m} / \mathrm{s}$ from that with no gust is large near $\phi \approx 0$, $\pi$. (2) Around $\phi \approx \pi$, the inflow velocity to the wing is increased by $w_{Y}$, and that around $\phi \approx 0$ is decreased. (3) In the present analysis, based on blade element theory, the lift and drag acting on the blade of a propeller around $\phi=0, \pi$ are nearly parallel to the $X-Y$ plane because the blade is nearly parallel to the $Z$ axis. Then, the lift and drag have a $Y$ component. Facts (1)-(3) cause the difference of $F_{Y P}(t)$ around $\phi \approx \pi$ and around $\phi \approx 0$. As stated above, the difference of $F_{Y P}(t)$ by one blade of one propeller causes $F_{Y P}$.

Figure 9 shows the variation in angle of attack of the flapping wings for case $\mathrm{A}$. The difference in $\alpha$ at $w_{Z}=$ $1 \mathrm{~m} / \mathrm{s}$ from that with no wind gust is large. However, the difference at $w_{Y}=1 \mathrm{~m} / \mathrm{s}$ from that with no wind gust is small. Moreover, the lift and drag acting on the flapping

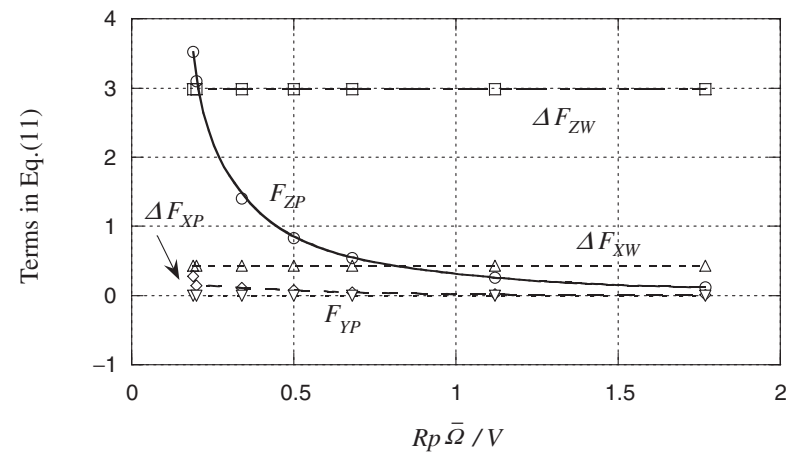

(b)

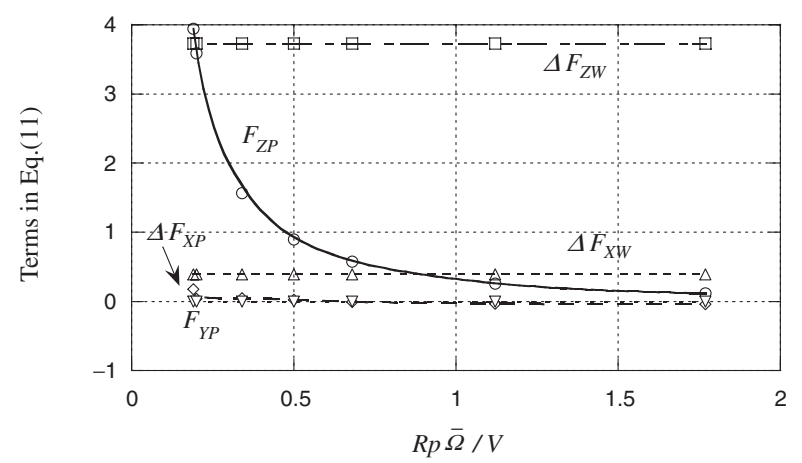

(d)
Fig. 4. Values of the terms in Eq. (11) for case B: (a) $w_{X}=1 \mathrm{~m} / \mathrm{s}$, (b) $w_{Z}=1 \mathrm{~m} / \mathrm{s}$, (c) $w_{Y}=1 \mathrm{~m} / \mathrm{s}$, (d) $w_{X}=w_{Z}=1 \mathrm{~m} / \mathrm{s}$ and (e) $w_{X}=w_{Y}=1 \mathrm{~m} / \mathrm{s}$.

wings are never parallel to the $Y$ axis because the flapping angle is not close to $\phi \approx \pm \pi / 2$. Therefore, the variation in $F_{Y}$ caused by $w_{Y}$ is not large. On the other hand, as stated above, the variation in propeller $F_{Y P}$ caused by $w_{Y}$ is large. This causes the $\Delta F_{\text {air }} / \Delta F_{\text {orn }}$ for $w_{Y}=1 \mathrm{~m} / \mathrm{s}$ at $R_{P} \bar{\Omega} / V=0.6$ (case A4) in Fig. 5(a) to be much larger than one.

The variation in moments around the $Y_{P}$ and $Z_{P}$ axes acting on one propeller (cases A1-2, B1-3) under $w_{Y}=1 \mathrm{~m} / \mathrm{s}$ and $w_{Z}=1 \mathrm{~m} / \mathrm{s}$, respectively, are not zero. ${ }^{11)}$ Variations of moments around the other axes under these wind gusts are zero. Furthermore, the variations of moments around the $X_{P}, Y_{P}$ and $Z_{P}$ axes are zero under $w_{X}=1 \mathrm{~m} / \mathrm{s}$. The moments of the two propellers cancel each other out as well as those of the right and left flapping wings. In order to estimate variations of moments around the $X_{B}, Y_{B}$ and $Z_{B}$ axes through the center of gravity of the airplane, these moments should be added to the moments due to the forces estimated above. 


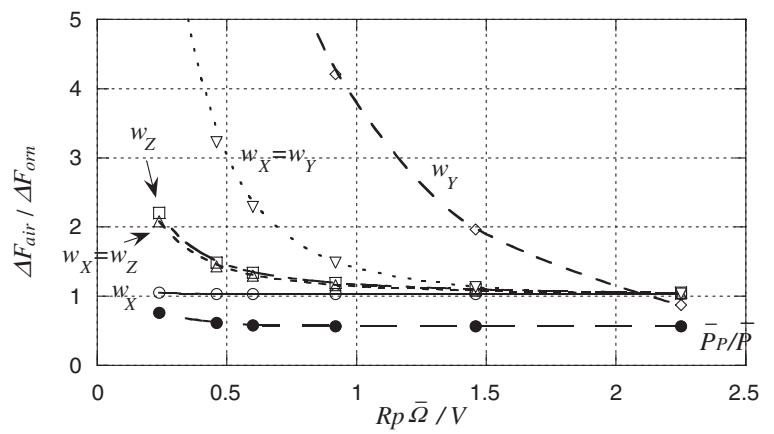

(a)

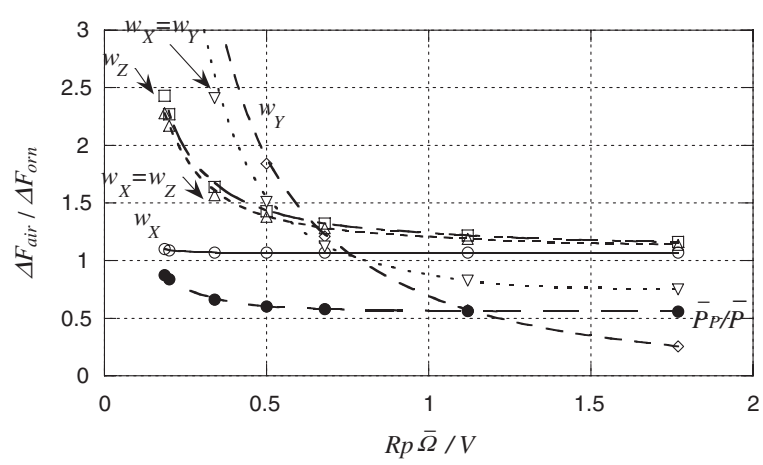

(b)

Fig. 5. Relationship between $R_{P} \bar{\Omega} / V$ and $\Delta F_{\text {air }} / \Delta F_{\text {orn }}$ : (a) case $\mathrm{A}$ and (b) case B.

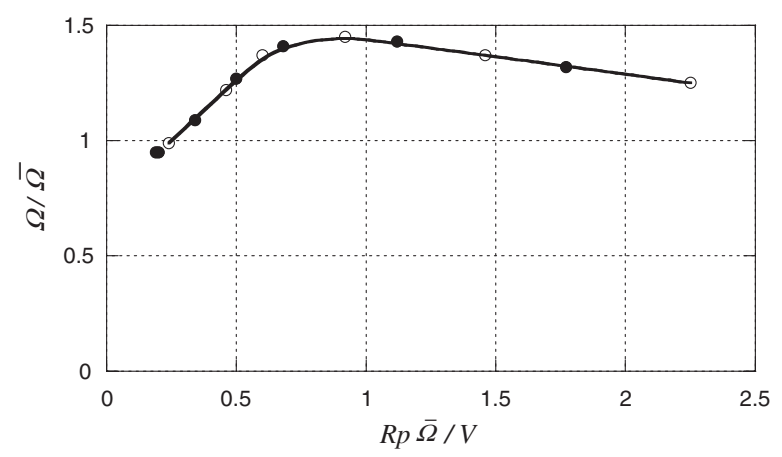

Fig. 6. Variation in flapping frequency of the ornithopter and rotational speed of the propeller. Open circles: case A1-6; Filled circles: case B1-7.

In the analysis so far, the consumed power was fixed when the ornithopter and the airplane encountered a wind gust. The variations of flapping frequency were small for all the wind gusts. Moreover, the variations of the rotational speed of the propellers of the airplane for the wind gusts $w_{Y}=1 \mathrm{~m} / \mathrm{s}, \quad w_{Z}=1 \mathrm{~m} / \mathrm{s}$ and $w_{Y}=w_{Z}=1 \mathrm{~m} / \mathrm{s}$ were small. Hereafter, the other analysis where the flapping frequency of the ornithopter and the rotational speed of propellers are fixed will be made when they encounter the wind gust $w_{X}=1 \mathrm{~m} / \mathrm{s}$. Table 6 shows the $F_{X}$ and $\Delta F_{\text {orn }}$ of the ornithopter. The values of $F_{Y}$ and $F_{Z}$ in this analysis are the same as those shown in Table 2. Figure 10 shows the values of $\Delta F_{\text {air }} / \Delta F_{\text {orn }}$ for the comparisons between case $A$ and case A1-6 and those between case B and case

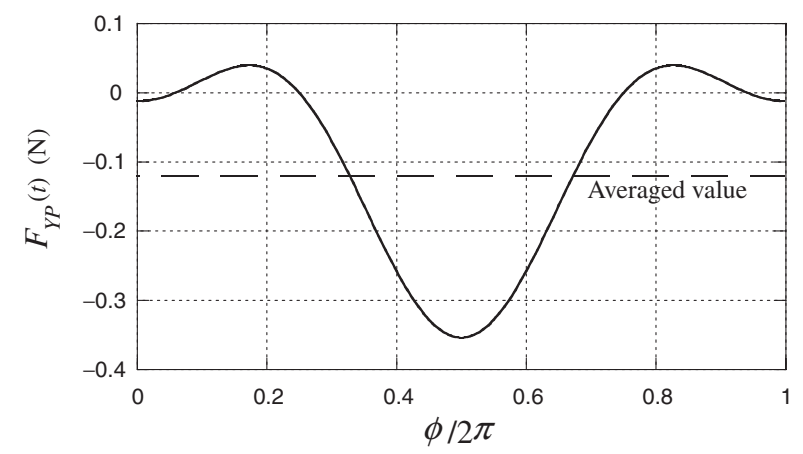

Fig. 7. Variation in $F_{Y P}$ generated by one blade of one propeller in case A4.

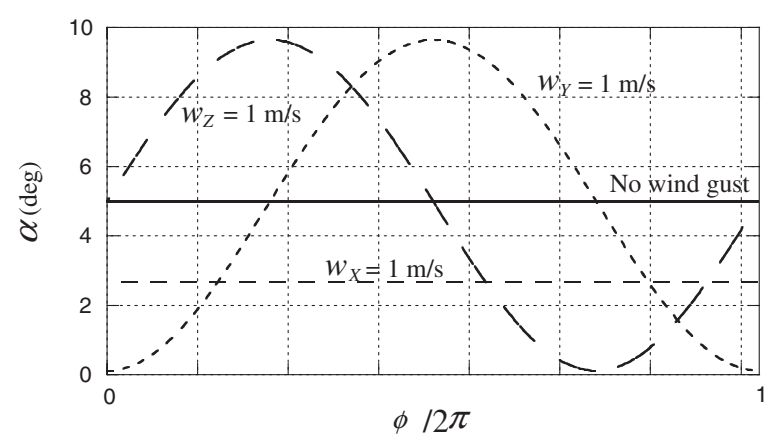

Fig. 8. Angle of attack of the propeller at the $75 \%$ spanwise position (case A4).

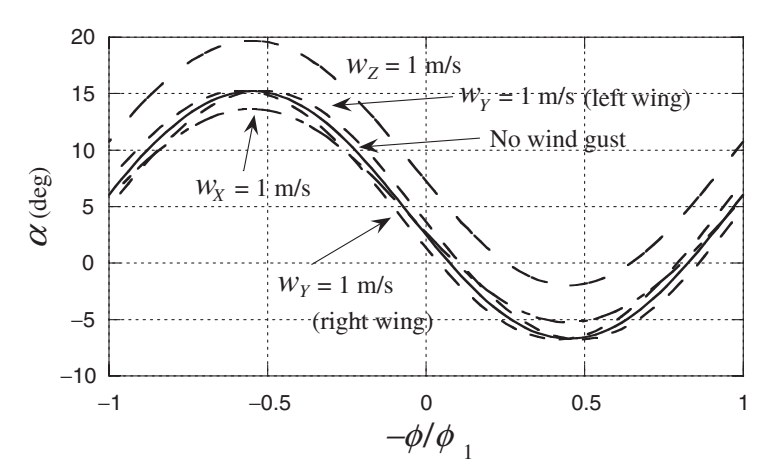

Fig. 9. Angle of attack of the flapping wing at the $75 \%$ spanwise position (case A).

B1-7. In this figure, the values of the horizontal axes are $R_{P} \bar{\Omega} / V$, and the values of $\Delta F_{\text {air }} / \Delta F_{\text {orn }}$. The values of $\bar{P}_{P} / \bar{P}$, which is a ratio of power between the airplane and ornithopter after they encounter the frontal wind gust, are also shown in this figure. The values of $\bar{P}_{P} / \bar{P}$ are less than one. The values of $\bar{P}_{P} / \bar{P}$ in this figure are less than those in Fig. 5 because the rotational speed for this figure is larger than that for Fig. 5. The values of $\Delta F_{\text {air }} / \Delta F_{\text {orn }}$ are always larger than one. The variation in aerodynamic force of the airplane with the propeller(s) is always larger than those of the ornithopters. This is an advantage of the ornithopters over the airplanes when they encounter frontal wind gust. This advantage is also observed under the condition of constant consumed power as shown in Figs. 5(a) and (b). 
Table 6. Aerodynamic force generated by the ornithopter encountering wind gust $w_{X}=1 \mathrm{~m} / \mathrm{s}$ (flapping frequency is fixed).

\begin{tabular}{lccc}
\hline & $F_{X}(\mathrm{~N})$ & $F_{X}-\bar{F}_{X}$ & $\Delta F_{\text {orn }}$ \\
\hline Case A & 0.286 & -0.046 & 0.492 \\
Case B & 0.128 & -0.043 & 0.472 \\
\hline
\end{tabular}

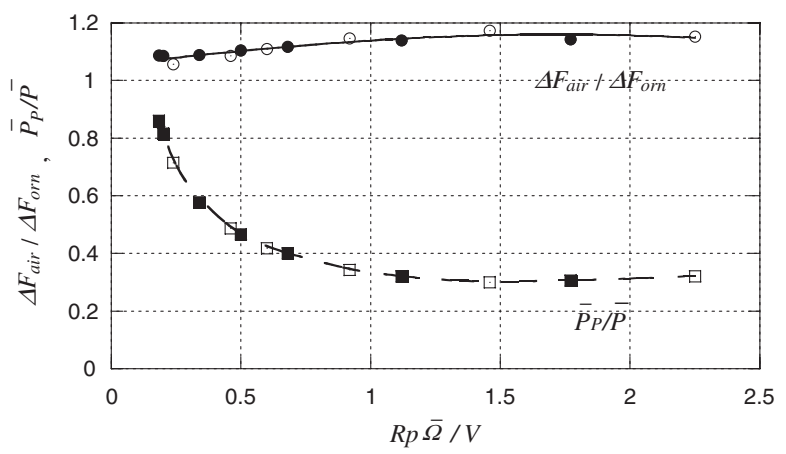

Fig. 10. Relationship between $R_{P} \bar{\Omega} / V$ and $\Delta F_{\text {air }} / \Delta F_{\text {orn }}, \bar{P}_{P} / \bar{P}$. Open circles: cases A and A1-6; Filled circles: cases B and B1-7. $\bar{P}_{P} / \bar{P}$ is a ratio of power between the airplane and ornithopter after they encounter frontal wind gust.

\section{Conclusions}

The variation in aerodynamic forces acting on an ornithopter and an airplane with one or two propellers, which generated the same thrust under no gust wind, was compared when they encountered a gust of wind. The analysis was based on blade element theory and momentum theory. The quasi-steady assumption was applied and unsteady effects were ignored.

Comparisons were made under the following two conditions. (Condition 1) The consumed power remained constant before and after they encountered a wind gust. (Condition 2) The period of one cycle of flapping motion and that of one rotation of propeller(s) remained constant. The conditions were nearly identical when they encountered side and verti- cal wind gusts. The following results were obtained under both conditions. (1) The variation in aerodynamic forces caused by vertical and frontal wind gusts of an ornithopter were a little smaller than those of an airplane with one or two propellers. (2) The difference in variation in aerodynamic forces caused by a side wind gust between them was often larger than that caused by vertical and frontal wind gusts. (3) The variation in aerodynamic forces caused by a side wind gust for the former was smaller than that for the latter when the reciprocal of the advance ratio of the propeller and the flapping amplitude of the ornithopter were small. Note that the tail wings of the airplane and interference between the main wing and propellers were ignored in the present analysis.

\section{References}

1) Taylor, G. K. and Thomas, A. L. R.: Dynamic Flight Stability in the Desert Locust Schistocerca gregaria, J. Exp. Biol., 206 (2003), pp. 2803-2829.

2) Sun, M. and Xiong, Y.: Dynamic Flight Stability of a Hovering Bumblebee, J. Exp. Biol., 208 (2005), pp. 447-459.

3) Sun, M. and Wang, J. K.: Flight Stabilization Control of a Hovering Model Insect, J. Exp. Biol., 210 (2007), pp. 2714-2722.

4) Faruque, I. and Humbert, J. S.: Dipteran Insect Flight Dynamics. Part 1: Longitudinal Motion about Hover, J. Theor. Biol., 264 (2010), pp. 538-552.

5) Faruque, I. and Humbert, J. S.: Dipteran Insect Flight Dynamics. Part 2: Lateral-Directional Motion about Hover, J. Theor. Biol., 265 (2010), pp. 306-313.

6) Orlowski, C. T. and Girard, A. R.: Dynamics, Stability, and Control Analyses of Flapping Wing Micro-Air Vehicles, Progress in Aerospace Sciences, 51 (2012), pp. 18-30.

7) Gao, N., Aono, H. and Liu, H.: Perturbation Analysis of 6DoF Flight Dynamics and Passive Dynamic Stability of Hovering Fruit Fly Drosophila melanogaster, J. Theor. Biol., 270 (2011), pp. 98-111.

8) Sigthorsson, D. O., Oppenheimer, M. W. and Doman, D. B.: Insect Sized Flapping Wing Vehicles versus Rotorcrafts, a Comparative Study, AIAA 2012-0028, 2012.

9) Sunada, S. and Tsuji, K.: Advantages of a Flapping Wing over a Propeller, AIAA 2012-0416, 2012.

10) Azuma, A.: The Biokinetics of Flying and Swimming, AIAA, Reston, Virginia, 2006.

11) Ribner, S. H.: Propellers in Yaw, NACA TR 820, 1943. 